

\section{Mauriac Syndrome in a Nigerian child with Type 1 Diabetes Mellitus: A Case Report Ezeani IU1, Oyenusi EE ${ }^{* 2}$}

${ }^{1}$ Endocrinology, Diabetes and Metabolism Unit, Department of Medicine, Federal Medical Centre Umuahia, Nigeria

${ }^{2}$ Endocrinology and Metabolism Unit, Department of Paediatrics, College of Medicine, University of Lagos/Lagos University Teaching Hospital, Lagos Nigeria

*Correspondence: Dr EE Oyenusi, P.O. Box 4337, Ikeja, Lagos, Nigeria. E-mail: eoyenusi@unilag.edu.ng; ORCID - https://orcid.org/0000-0003-1314-5236.

\section{Summary}

A 14-year-old boy with Type 1 Diabetes mellitus (diagnosed at eight years of age) presented with complaints of fever, weight loss, growth failure, pubertal delay, abdominal swelling and discomfort. He was on Premixed insulin (70/30) with inadequate follow-up and poor diabetic control. Examination revealed cachexia, generalised lymphadenopathy, a protuberant abdomen and hepatosplenomegaly. Anthropometry showed a bodyweight of $19.6 \mathrm{~kg}$, a height of $116 \mathrm{~cm}$ and a BMI of $14.1 \mathrm{~kg} / \mathrm{m}^{2}$, all markedly below the $3^{\text {rd }}$ centile. He had no secondary sexual characteristics: axillary hair stage 1, pubic hair stage 1, penile length of $4.9 \mathrm{~cm}$ and prepubertal testicular volumes of $3 \mathrm{mls}$ bilaterally. At presentation, his random blood glucose was $400 \mathrm{mg} / \mathrm{dl}$, and glycosylated haemoglobin was $11.6 \%$. Screening for tuberculosis, human immunodeficiency virus, hepatitis and lymphoproliferative disorders were negative. Other blood investigation findings included leucocytosis, erythrocyte sedimentation rate of $30 \mathrm{~mm} / \mathrm{hr}$, normal liver function tests, normal serum electrolytes, urea and creatinine. His haemoglobin genotype was AS. Chest radiograph showed features of bronchopneumonia. A presumptive diagnosis of Mauriac Syndrome was made. With the optimisation of glycaemic control, he improved clinically with a weight gain of $5.7 \mathrm{~kg}$ over four months and resolution of hepatosplenomegaly.

Keywords: Abdominal swelling, Cachexia, Growth failure, Hepatomegaly, Insulin, Pubertal delay.

\section{Introduction}

Mauriac syndrome (MS) is characterised by growth failure, delayed puberty, cushingoid features and hepatomegaly, usually associated with poor control of Type 1 Diabetes mellitus (T1DM) in children and adolescents. ${ }^{[1-4]}$ The pathophysiology of MS is not entirely understood, but some mechanisms for glycogenosis have been suggested. In prolonged hyperglycaemia, glucose passively enters the hepatocytes by the insulinindependent membrane glucose transporter
GLUT2 mechanism and is rapidly phosphorylated. Subsequent insulin treatment promotes the polymerisation of glucose-6phosphate to glycogen by glycogen-synthase, driving a large amount of glycogen synthesis in the presence of trapped high cytoplasmic glucose concentrations. [1,2,5-7] Some authors have suggested that genetic factors may be involved in the predisposition to MS, which may explain why some patients with poorly controlled T1DM develop the condition while others do not. [1,8] 
MacDonald et al. hypothesised that a heterozygous mutation found in a subunit of the liver glycogen phosphorylase kinase enzyme complex (which catalyses the first step in glycogen breakdown in the liver) combined with extreme hyperglycaemia acted together to inhibit glycogenolysis and to cause massive glycogen accumulation in the liver cells. ${ }^{[8]}$ The glycogen deposition in the liver described in MS has also been noted in other reports without the full spectrum of MS, both in adults and children with T1DM and rarely in T2DM. [9] Some of the different terminologies used to describe this entity include hepatic glycogenosis, glycogen hepatopathy, glycogen storage hepatomegaly and hepatic glycogen storage. [9] Torbenson et al. ${ }^{[10]}$ first used the term "glycogenic hepatopathy" to describe their histopathological findings in 14 children and adults with the condition. This term has been used universally since then to describe this unique pathologic feature. $[9,10]$

No sex preponderance has been noted in the literature. [1-4]. Current thinking is that a diagnosis of MS can be made based on clinical features and the results of imaging and laboratory tests associated with good clinical response, while liver biopsy can be reserved for unclear diagnosis or non-response. [1,2,4] Timely institution of good glycaemic control can reverse these features with no case of evolution to end-stage liver disease. ${ }^{[1-4]}$ This report is presented to emphasise the need to maintain a high index of suspicion for prompt diagnosis and management.

\section{Case Description}

A 14-year-old boy with T1DM who was diagnosed at eight years of age presented to the Endocrinology Clinic of the Federal Medical Centre, Umuahia, in May 2020, with complaints of fever, weight loss and pubertal delay. There was associated abdominal swelling and discomfort. The only significant previous history was jaundice in the neonatal period, which resolved within two weeks. Initial diagnosis of DM was made when he presented in diabetic ketoacidosis and was managed with intravenous insulin. He was subsequently placed on subcutaneous pre-mixed (70/30) insulin (1.42units/kg), but his compliance was poor due to severe financial constraints and an irregular supply of insulin. Clinic attendance was also erratic because of low finances and distance from his rural abode to the health facility. He could not afford a home blood glucose monitoring device due to poverty. $\mathrm{He}$ was not covered by medical insurance, and his medical bills were being footed by donations.

On examination, he was cachectic, anicteric, afebrile, not pale but had generalised lymphadenopathy. There were no peripheral stigmata of chronic liver disease. He had a protuberant abdomen, hepatomegaly $(4 \mathrm{~cm}$ below the right costal margin) and splenomegaly $(3 \mathrm{~cm}$ below the left costal margin). Anthropometry showed a bodyweight of $19.6 \mathrm{~kg}$, height of $116 \mathrm{~cm}$ and Body Mass Index (BMI) $14.1 \mathrm{~kg} / \mathrm{m}^{2}$, all markedly below the third percentile for age and sex. There were no secondary sexual characteristics such as axillary hair Stage 1, pubic hair Stage 1, penile length of $4.9 \mathrm{~cm}$ and prepubertal testicular volumes of $3 \mathrm{mls}$ bilaterally (Figure 1).

The results of laboratory evaluation at presentation included a random blood glucose of $400 \mathrm{mg} / \mathrm{dl} \quad(22.2 \mathrm{mmol} /)$, glycosylated haemoglobin (HbA1c) level of $11.6 \%$ $(103 \mathrm{mmol} / \mathrm{mol})$ [higher than the target for good glycaemic control of $<6.5 \%$ $(58.5 \mathrm{mmol} / \mathrm{mol})]$. Others included a Packed Cell Volume (PCV) of $34.7 \%$, haemoglobin concentration of $12.5 \mathrm{~g} / \mathrm{dl}$, White Blood Cell count (WBC) of $11,870 / \mathrm{mm}^{3}$ (neutrophils $54.5 \%$, lymphocytes $-30 \%$ ), platelet count of $338,000 / \mathrm{mm}^{3}$. Erythrocyte Sedimentation Rate was $30 \mathrm{~mm} / \mathrm{hr}$. The haemoglobin genotype was AS. Serum electrolytes were normal: bicarbonate -24.5 , sodium - 132 , chloride -97 , potassium - 4.3, (all in $\mathrm{mmol} / \mathrm{L}$ ); urea $22 \mathrm{mg} / \mathrm{dl} \quad(10-55 \mathrm{mg} / \mathrm{dl})$ and creatinine - 
$0.3 \mathrm{mg} / \mathrm{dl}$ (male: $0.7-1.2 \mathrm{mg} / \mathrm{dl}$ ). Liver function tests (LFTs) were normal: alanine transaminase (ALT) -10.4 (up to 41U/1), aspartate transaminase (AST) - 15.4 (up to 40U/1), alkaline phosphatase (ALP) - 87.3 (40-130U/1), Gamma Glutamyltransferase (GGT): 42.4
(<60U/1), Total bilirubin $0.3(0.2-1.0 \mathrm{mg} / \mathrm{dl})$, direct - 0.10 (up to 0.2 ), indirect 0.2 (up to $0.8 \mathrm{mg} / \mathrm{dl}$ ). Screening for hepatitis B and C were negative.

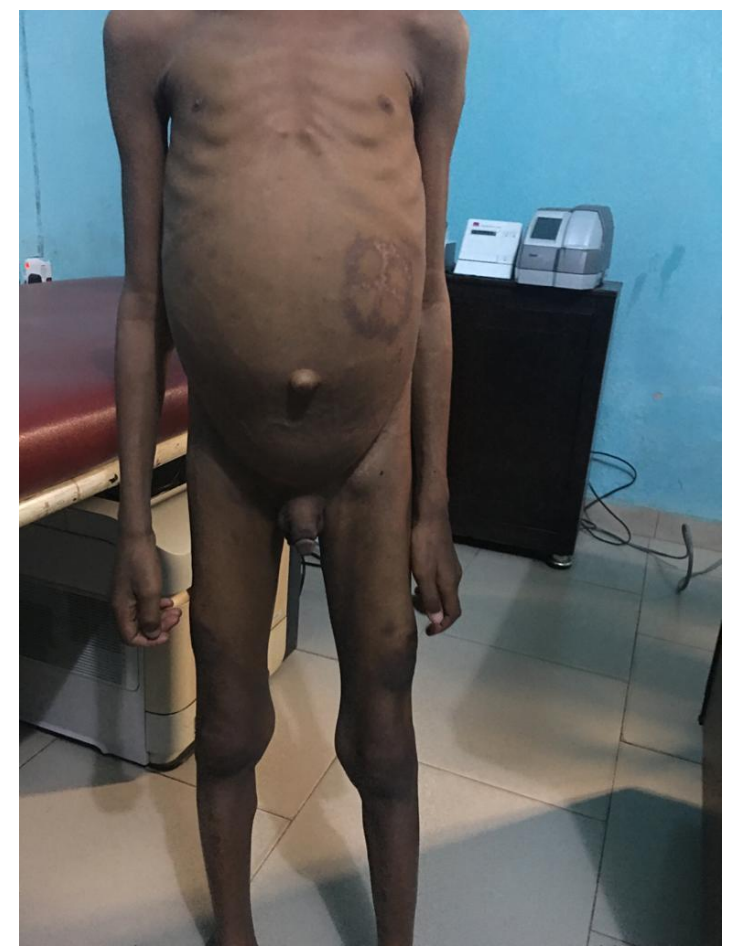

Figure 1: Patient at presentation [showing short stature, cachexia and absent secondary sexual characteristics]

Chest radiograph showed patchy opacities suggestive of bronchopneumonia but not tuberculosis. GeneXpert and Human Immunodeficiency Virus (HIV) screening were negative. Initial screening for lymphoproliferative disorders with peripheral blood film was normal. Abdominal ultrasound scan (USS) findings included minimal hepatomegaly with preserved parenchymal echogenicity, splenomegaly with preserved echotexture, normal dimensions of kidneys with good corticomedullary distinction, normal gall bladder, pancreas, adrenals, and abdominal aorta; no masses, free fluid or dilation of bowel loops was seen. Due to financial constraints, serum lipids, bone marrow aspiration, lymph node, and liver biopsy could not be done. A presumptive diagnosis of Mauriac syndrome was made based on his clinical and obtainable laboratory findings.

Available resources were channelled towards optimising the blood glucose control with accessible and affordable insulin, premixed in the ratio of $70 / 30$. He was also treated with intravenous antibiotics (ceftriaxone) for five days and discharged on oral ciprofloxacin for seven days. The blood glucose control improved, and the patient gained $5.7 \mathrm{~kg}$ over four months with a resolution of the hepatomegaly and splenomegaly (Figure 2). He is yet to develop any secondary sexual characteristics. He is on follow-up care, and efforts are being made to get him on sustainable NGO support for his insulin supplies and blood glucose testing kits. 


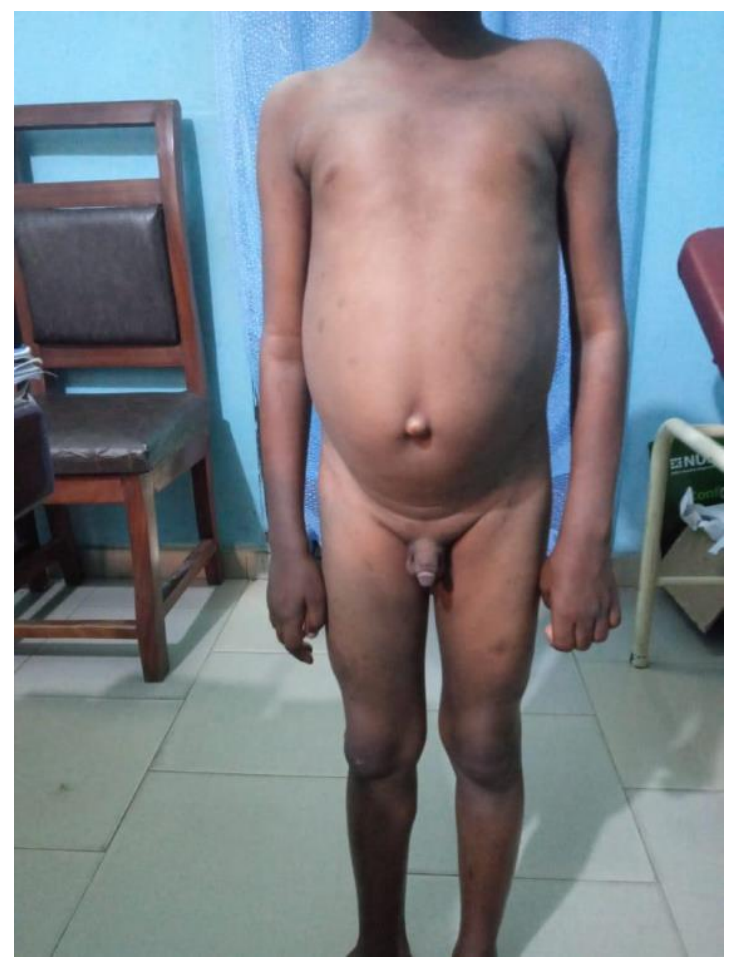

Figure 2: Four months after treatment [showing no cachexia]

\section{Discussion}

Mauriac syndrome was originally described in 1930 as a triad of poorly controlled diabetes mellitus, hepatomegaly and short stature. ${ }^{[1,9]}$ Over time, the definition has been expanded to include other variable features such as delayed puberty, cushingoid habitus and growth failure. $[1-3,5]$ The index patient presented with hepatomegaly, growth failure and pubertal delay on a background of poorly controlled diabetes mellitus as evidenced by the high level of $\mathrm{HbA1C}$ and hyperglycaemia at presentation.

The peak age of occurrence of MS is in adolescence. The age of the index patient fits into the age incidence. A retrospective study by Fitzpatrick et al. [2] of about 31 patients reported a median age of 15.1 years (IQR 14-16.2). Similar age brackets ranging from 11-19 years have been documented in various case reports. [1,3,4,11-13] Adolescence is critical for T1DM patients with poor compliance. Even in climes with more flexible and readily available insulin regimens, adolescence still presents the paediatric diabetologist with significant management difficulties because of multiple factors, including conflict between an emerging individual identity and the control of carers and behavioural peculiarities of this age group. ${ }^{[1,4,5]}$ The low socioeconomic status of our patient and lack of access to a flexible insulin regimen could have contributed to suboptimal management of his condition. A case report from Sudan by Omer documented a background similar to that of the index patient and the use of premixed insulin. [13]

As observed in previous reports, the index patient was severely stunted and wasted with absent secondary sexual characteristics. [1,13,14] The growth failure observed in some patients with MS is evident in the suboptimal anthropometric parameters. Studies have reported height-for-age $z$-scores ranging from 1.01 to 0.4 and BMI-for-age $z$-scores of -12 to 0.67. [2, 13] Poor glycaemic control was also present with the high $\mathrm{HbA1C}$, similar to other reports. [1,2, 13] The index patient had no derangement of hepatic enzymes in agreement 
with previous reports. [1,2,5,13] The consequent liver damage from glycogen deposition usually, though not always, leads to an increase in the serum hepatic transaminases but with preservation of the synthetic function of the liver. ${ }^{[1,5,7]}$ splenomegaly has been reported in $16 \%$ of children with MS [2] and two patients in another report by Munns et al. [4] though this finding is yet unexplained. The splenomegaly observed in the index patient could have resulted from the associated acute infection suggested by leucocytosis and pneumonic changes on the chest radiograph. [15]

The diagnosis of MS is made from the typical clinical features and exclusion of other differential diagnoses. Serum levels of antinuclear antibodies, anti-smooth muscle antibodies, antimitochondrial antibodies and antineutrophil cytoplasmic antibodies are used to exclude autoimmune hepatitis. ${ }^{[1]}$ Serological tests to rule out infectious causes of hepatomegaly such as Epstein Barr virus, cytomegalovirus, hepatitis A virus, hepatitis B virus, hepatitis $\mathrm{C}$ virus, human immunodeficiency virus can also be undertaken. In cases of suspicion of haemochromatosis, serum iron studies should be done while serum copper/ceruloplasmin and a urinary penicillamine challenge are used to exclude Wilson disease. ${ }^{[1,2]}$ To investigate other causes of growth failure seen in these patients, haemoglobin genotype can be used to rule out sickle cell anaemia, especially in susceptible populations like our setting. Abdominal ultrasound is a simple and readily available tool to determine the dimension and characteristics of the liver tissue and rule out other pathology in organs such as pancreatitis. [1] Given some of the clinical features in the index patient, malnutrition-modulated diabetes was considered. The absence of pancreatic calcifications on abdominal USS and the initial mode of presentation in ketoacidosis of the index patient at the diagnosis of DM makes this differential diagnosis unlikely. [16]
Thyroid Function Tests, serologic screening for coeliac disease and serum insulin-like growth factor 1 (IGF1) can be carried out if the clinical features suggest these conditions. Financial constraints in the index patient prevented screening for coeliac disease using the assay for tissue transglutaminase antibody and antiendomysial antibody. However, the absence of other clinical features such as nausea, vomiting, diarrhoea, hypoglycaemia and no haematological evidence of hypochromic microcytic anaemia makes coeliac disease unlikely. [17] Relevant radiographs can determine skeletal age. Radiological imaging studies like computed tomography and magnetic resonance imaging in establishing the diagnosis of MS could depend on the interval changes in the liver density, but their sensitivity and specificity are yet to be ascertained. [1]

Non-alcoholic fatty liver disease (which may have a potential for significant liver disease in adulthood) share some features such as steatosis, significant dyslipidaemia and ultrasonographic liver appearance with MS. [2] This condition was unlikely in the index patient because children with non-alcoholic fatty liver disease are predominantly overweight/obese. In contrast, children with MS are thin, as seen in the index patient. As autoimmune hepatitis can be associated with T1DM, liver biopsy is usually undertaken when this or an alternate diagnosis to MS is suspected, especially in children with significantly abnormal transaminases (>twice the upper limit of normal). ${ }^{[2]}$ Given this, a liver biopsy may not be necessary for the index patient because his transaminases were normal.

Limitations: Some essential investigations required to exclude other causes of autoimmune hepatitis and growth failure in the index patient could not be done due to severe financial constraints. A more extended period of follow-up would have also enriched the report.

\section{Conclusion}


MS is a rare complication of poorly controlled T1DM, and a high index of suspicion should be maintained for prompt diagnosis. Timely intervention with good glycaemic control reverses the clinical features in most cases, and adequate growth is achieved.

Acknowledgement: The authors wish to thank the patient and his parents for their consent and permission to use the patient's information and photographs for this report.

Authors' Contributions: EIU and OEE conceived the research and gathered the data. OEE did the literature review and manuscript drafting. Both authors revised the manuscript for sound intellectual contents and approved the final version of the manuscript.

Conflicts of Interest: None.

Funding: Self-funded.

Publication History: Submitted 07 October 2021; Accepted 13 November 2021.

\section{References}

1. Lombardo L, Passanisi S, Gasbarro A, Tuccari G, Leni A, Salzano G. Hepatomegaly and type 1 diabetes: a clinical case of Mauriac's syndrome. Ital J Paediatr 2019; 45: 1-6. https://doi.org/10.1186/s13052-018-05982

2. Fitzpatrick E, Cotoi C, Quaglia A, Sakellariou S, Ford-Adams ME, Hadzic N. Hepatopathy of Mauriac syndrome: a retrospective review from a tertiary liver centre. Arch Dis Child 2014; 99: 354-357. http://dx.doi.org/10.1136/archdischild2013-304426

3. Kocova M, Milenkova L. Old syndromenew approach: Mauriac syndrome treated with continuous insulin delivery. SAGE Open Med Case Rep. 2018; 6: 1-4. https://doi.org/10.1177/2050313X1878551 $\underline{0}$

4. Munns CFJ, Mccrossin RB, Thomsett MJ, Batch J. Hepatic glycogenosis: Reversible hepatomegaly in type 1 diabetes. J Paediatr
Child Health 2000; 36: 449-452.

https://doi.org/10.1046/j.1440$\underline{1754.2000 .00547 . x}$

5. Elder CJ, Natarajan A. Mauriac syndrome-a modern reality. J Pediatr Endocrinol Metab 2010; 23: 311-313. https://doi.org/10.1515/jpem.2010.23.3.31 $\underline{1}$

6. Ferrer JC, Favre C, Gomis RR, FernándezNovell JM, García- Rocha M, de la Iglesia N, et al Control of glycogen deposition. FEBS Lett 2003; 546: 127-132. https://doi.org/10.1016/S00145793(03)00565-9

7. Chatila R, West AB. Hepatomegaly and abnormal liver tests due to glycogenosis in adults with diabetes. Medicine 1996; 75: 327-33.

https://doi.org/10.1097/00005792199611000-00003

8. MacDonald MJ, Hasan NM, Ansari IU, Longacre MJ, Kendrick MA, Stoker SW. Discovery of a genetic metabolic cause for Mauriac syndrome in type 1 diabetes. Diabetes. 2016; 65: 2051-2059. https://doi.org/10.2337/db16-0099

9. Sherigar JM, Castro J, Yin YM, Guss D, Mohanty SR. Glycogenic hepatopathy: A narrative review. World J Hepatol 2018; 10:172-185.

https://doi.org/10.4254/wih.v10.i2.172

10. Torbenson $M$, Chen $Y Y$, Brunt E, Cummings OW, Gottfried M, Jakate S, et al. Glycogenic hepatopathy: an underrecognised hepatic complication of diabetes mellitus. Am J Surg Pathol 2006; 30 (4): 508513. https:// doi.org/10.1097/00000478200604000-00012

11. Gutch M, Philip R, Saran S, Tyagi $R$, Gupta KK. Re-emergence of a rare syndrome: A case of Mauriac syndrome. Indian J Endocrinol Metab 2013; 17: S283S285. https://doi.org/10.4103/2230$\underline{8210.119611}$

12. Imtiaz KE, Healy C, Sharif S, Drake I, Awan F, Riley J, et al. Glycogenic 
hepatopathy in type 1 diabetes: an underrecognised condition. Diabetes Care 2013; 36: e6-e7. https://doi.org/10.2337/dc12$\underline{1134}$

13. Omer IM. Mauriac Syndrome: A diagnosis that still exists. Acad J Ped Neonatol 2017; 3: https://doi.org/10.19080/AJPN.2017.03.5 $\underline{55647}$

14. Elamin A, Aitahir H, Ismail B, Tuvemo I. Clinical pattern of childhood Type 1 (insulin-dependent) diabetes mellitus in Sudan. Diabetologia 1992; 35: 645-648. https://doi.org/10.1007/BF00400256

15. Suttorp M, Classen CF. Splenomegaly in Children and Adolescents. Front Pediatr
2021;

9:

704635.

https://doi.org/10.3389/fped.2021.704635

16. Haftu H, Gebrearegay H, Berhane A. Malnutrition-Modulated Diabetes Mellitus in Children, Rare Disease with Atypical Presentation: Case Report. Diabetes, Metab Synd Obesity Targets Therapy 2020; 13: 3069-3074.

https://doi.org/10.2147/DMSO.S263229

17. Mahmud FH, Elbarbary NS, FröhlichReiterer E, Holl RW, Kordonouri O, Knip M et al. ISPAD Clinical Practice Consensus Guidelines 2018: Other complications and associated conditions in children and adolescents with type 1 diabetes. Pediatr Diabetes 2018; 19: 275-286. https://doi.org/10.1111/pedi.12740 original source is adequately cited and credited. 